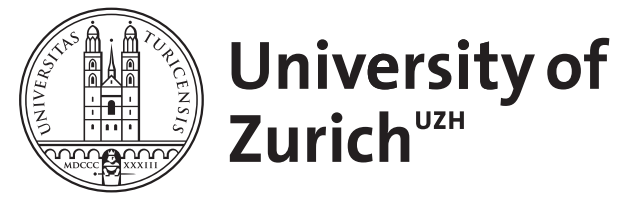

Zurich Open Repository and Archive

University of Zurich

University Library

Strickhofstrasse 39

CH-8057 Zurich

www.zora.uzh.ch

Year: 2019

\title{
Patient drop-outs in clinical trials
}

Papageorgiou, Spyridon N

DOI: https://doi.org/10.1177/1465312519883891

Posted at the Zurich Open Repository and Archive, University of Zurich

ZORA URL: https://doi.org/10.5167/uzh-186067

Journal Article

Accepted Version

Originally published at:

Papageorgiou, Spyridon N (2019). Patient drop-outs in clinical trials. Journal of Orthodontics, 46(4):378-380.

DOI: https://doi.org/10.1177/1465312519883891 


\section{Patient drop-outs in clinical trials}

Spyridon N. Papageorgiou ${ }^{1}$

${ }^{1}$ Clinic of Orthodontics and Pediatric Dentistry, Center of Dental Medicine, University of Zurich, Zurich, Switzerland

Corresponding author: Spyridon N Papageorgiou, Clinic of Orthodontics and Pediatric Dentistry, Center of Dental Medicine, University of Zurich, Plattenstrasse 11, Zurich $\mathrm{CH}$ 8032, Switzerland.

Email: snpapage@gmail.com.

Words in text: 1647

\section{Declaration of conflicting interests}

The author(s) declared no potential conflicts of interest with respect to the research, authorship, and/or publication of this article.

\section{Funding}

The author(s) received no financial support for the research, authorship, and/or publication of this article.

\section{ORCID ID}

Spyridon N Papageorgiou https://orcid.org/0000-0003-1968-3326 


\section{Theoretical scenario}

A group of orthodontists plan to run a clinical trial to compare the alignment duration using three different kinds of fixed appliances. Group A will receive conventional $0.0186 \times 0.025$-inch straight-wire appliances with a standard archwire sequence ligated with conventional elastomeric ligatures. Group B will receive the same fixed appliance plus the application of low level laser therapy at every appointment. Group $\mathrm{C}$ will the receive the same fixed appliance as Group A plus the application of corticotomies at every appointment. The trial's primary endpoint is to be the duration of the alignment phase of fixed appliance treatment, which will be calculated as the sum of days needed from appliance insertion until a rectangular $0.0166 \times 0.022$ inch stainless steel archwire can be passively inserted in the slots of all brackets.

The authors want their trial to be adequately-powered to provide a definitive answer to the research null hypothesis that there is no difference in the alignment duration among the three appliance groups evaluated. Therefore, they conduct an a priori sample size calculation, using as reference a previous trial (Ong et al., 2011), which they judge to be set similarly to their own. The expected value for Group A is taken from that reference trial to be 132.0 days (standard deviation of 36.0 days) and they hypothesize a $10 \%$ reduction (118.8 days) and a 20\% reduction (105.6 days) in the alignment duration of Groups B and C, respectively (with a common standard deviation of 36.0 days). They aim to use a one-way analysis of variance to analyse the data and set the significance level (type I error rate) at $5 \%$, while aiming for power of $90 \%$ (i.e. 1 minus a 10\% type II error rate). This sample size calculation indicates that 49 patients/group would be needed (147 patients in total), which is increased to a recruitment goal of 57 patients/group (171 patients in total) to account for an expected 15\% drop-out rate.

The clinical trial is initiated according to its registered protocol and a total of 171 patients are allocated with a 1:1:1 allocation ratio in the three groups (57 patients/group). After administering the planned interventions, the patients are followed up until trial completion when the same stainless steel working archwire is placed passively in all patients. At this point outcome data is collected from 150 patients in total,

as several patients dropped out of the trial and no outcome data was available. The reasons for these 26 missing patients were: discontinuation due to poor oral hygiene or poor compliance (6 patients), relocation (5 patients), pain or discomfort (15 patients). 
The authors analyse the data and find no statistically significant difference in the alignment duration among the three groups, concluding that their trial provided robust evidence about the similar clinical performance of the three groups.

\section{Which of the following statements are correct, if any:}

(A) The current trial is adequately powered to identify the hypothesised difference in alignment duration, since a sample size calculation was performed.

(B) The current trial might be adequately powered to identify the hypothesized difference in alignment duration, since a sample size calculation was performed, and drop-outs were less than the expected $15 \%$ (26 drop-outs / 171 patients 15\%).

(C) Estimations about the alignment duration in each of the three groups of the trial can be done with similar precision, since patients in the trial were randomized with a 1:1:1 allocation ratio.

(D) The fact that the drop-outs were not similar across the three groups introduces de facto bias in the trial's results.

(E) The uneven drop-out rate across the three groups might introduce bias in the trial's results, if the reasons for the drop-outs are related to the administered intervention.

\section{Discussion}

The subject of a trial's statistical power and how it relates to sample size calculation has been somewhat discussed in previous issues (Papageorgiou 2019a,b). The current trial included a sample size calculation aiming to identify a $10 \%-20 \%$ reduction in alignment duration with $90 \%$ power, which indicated that at least 49 patients/group (147 in total) would be needed to be analysed. Therefore, if the hypothesised difference in alignment duration (10\%-20\%) or one larger than that exists in reality, then the current trial would have adequate power (here, interpreted as $90 \%$ power) to identify this. This, of course, holds true only on the condition that the originally planned sample of 49 patients/group (147 in total) are included and finally analysed at the trial's conclusion. So statement $(A)$ does not necessarily hold true and performing an a priori sample size calculation is a necessary, but not adequate, step in ensuring a trial is adequately powered. 
It is however generally accepted that in most clinical trials are prone to some loss of participants and therefore data that can be eventually analysed. These patient "drop-outs" can occur in different phases of a clinical trial and be of variable magnitude - especially in clinical trials with prolonged follow-up durations of many years. For example, a patient that has been randomised to one group might fail to attend the next appointment, where treatment will be administered. Similarly, a patient might not be available for any of the following appointments, where treatment is continued or the patient is simply observed. Usually though, drop-out rates of $10 \%-20 \%$ are considered moderate and might not necessarily be an issue to the trial's credibility, provided they do not overly reduce the sample being available for analysis. This is also the reason why many trialists take into account during the planning stage of the protocol the expected patient drop-outs in their sample size calculation. Simply put, the required sample to be recruited is inflated by the expected drop-out rate so that the sample after drop-outs still that is finally analysed has adequate statistical power. In the current theoretical example the overall drop-out rate was in the average magnitude that was expected and incorporated (15\%) and we might reasonably assume that the trial might have adequate statistical power to identify the target effect size (if this really exists). This supports statement (B).

Patient drop-outs during a trial are generally to be expected in clinical research and might not be detrimental to a trial's credibility - provided they are of small to moderate and occur at random. The occurring at random can be interpreted as (i) drop-outs being of the same magnitude more across all randomised groups, (ii) drop-outs occurring at the similar phases of treatment, and (iii) lack of any systematic pattern that dictates patient drop-outs. Points (i) and (ii) are of particular interest, since drop-outs decrease the analysed sample in each group and through this the statistical analysis. Looking at the theoretical example of this trial we can see that Group A and Group B have a larger number of analysed patients that Group C (51 patients compared to 43 patients), which contradicts the 1:1:1 initial allocation ratio. This in turns means that we run the risk of observing larger standard deviations in Group C than the other two groups, which might not be related to a more heterogeneous clinical treatment response, but might be due to sampling reasons. Consequently, this might translate to greater imprecision in the effect estimation compared to the other two groups that might not enable us to identify an existing difference. Therefore, statement $\mathrm{C}$ is not true.

Finally, when interpreting the results of a clinical trial, it is very important to know and critically appraise the reasons for any observed drop-outs in order to ascertain that no systemic pattern exists. 
Several reasons might exist for an initially randomised patient not being analysed in the end. The reasons for a patient missing on a planned appointment might be for personal reasons that have little to do with the treatment, like the patient moving to a different location, the patient being ill, or just not motivated enough. Similarly, trial outcome data might be missing randomly in the end due to the dental casts or radiographs being lost or of low quality. These drop-outs cannot be predicted by a single factor and are thought to be missing at random and not introduce bias in the trial's results (so statement $D$ is false). On the other side, patients might be prone to miss an oncoming appointment or discontinue treatment completely if the administered treatment (i) does not work as expected and/or (ii) is associated with adverse effects to the patient. In this case it might be possible that reduced efficacy or disproportionate harms have influenced the comparability of the analysed sample, which introduces bias in the trial's results since the sample doesn't any more represent the average recruited patient. In the present theoretical example, the trialists meticulously noted and reported the reasons for patient drop-outs in Table 1.

Table 1. Patient drop-outs during the trial with details.

\begin{tabular}{|l|l|l|l|l|}
\hline Status & Total & Group A & Group B & Group C \\
\hline Recruited & 171 & 57 & 57 & 57 \\
\hline & & & & \\
\hline Drop-outs: poor hygiene / compliance & 6 & 3 & 2 & 1 \\
\hline Drop-outs: relocated & 5 & 2 & 2 & 1 \\
\hline Drop-outs: pain / discomfort & 15 & 1 & 2 & 12 \\
\hline Drop-outs (total) & 26 & 6 & 6 & 14 \\
\hline & & & & \\
\hline Analysed & 145 & 51 & 51 & 43 \\
\hline
\end{tabular}

It is immediately obvious that drop-outs due to poor oral hygiene / compliance and relocations are relatively similar across the three groups. On the otherside, the vast majority $(80 \%)$ of drop-outs due to patientreported adverse effects (pain / discomfort) are found in Group C. If one also considers that Group C received the most invasive surgical procedure of the three groups, it might be reasonably to assume that the administered corticotomies could have been followed by adverse effects like bleeding, inflammation, pain, etc. This is important information that must be meticulously reported in the trial's report according to the CONSORT (Consolidated Standards of Reporting Trials) statement (Moher et al., 2010) in order to be able to fairly appraise the clinical performance of an intervention, but is often under-reported (Harrison 2003). Apart from that however, excluding patient drop-outs from the analysis might introduce bias in the 
trial's results, since a particular part of the original sample is missing and the groups are not comparable anymore. Therefore, statement $(E)$ is true. In such cases, advanced statistical methods (Unnebrink and Windeler, 2001) might be needed in order to impute the missing patients and reduce the risk of bias.

\section{References}

Harrison JE (2003) Clinical trials in orthodontics II: assessment of the quality of reporting of clinical trials published in three orthodontic journals between 1989 and 1998. Journal of Orthodontics 30: 309315.

Moher D, Hopewell S, Schulz KF, Montori V, Gøtzsche PC, Devereaux PJ, Elbourne D, Egger M, Altman DG, and Consolidated Standards of Reporting Trials Group (2010) CONSORT 2010 Explanation and Elaboration: Updated guidelines for reporting parallel group randomised trials. Journal of Clinical Epidemiology 63: e1-37.

Ong E, Ho C and Miles P (2011) Alignment efficiency and discomfort of three orthodontic archwire sequences: a randomized clinical trial. Journal of Orthodontics 38: 32-39.

Papageorgiou SN (2019a) Planning and interpreting the sample size of trials with multiple outcomes. Journal of Orthodontics 46: 74-76.

Papageorgiou SN (2019b) Clinically relevant sample size calculations for trials. Journal of Orthodontics 46: $178-180$.

Unnebrink K, Windeler J. Intention-to-treat: methods for dealing with missing values in clinical trials of progressively deteriorating diseases. Stat Med. 2001 Dec 30;20(24):3931-46. 